\title{
High-precision collinear laser spectroscopy at the Collinear Apparatus for Laser Spectroscopy and Applied Physics (COALA)
}

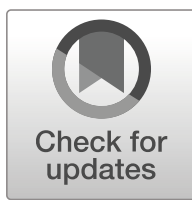

\author{
Phillip Imgram ${ }^{1}$ (D) . Kristian König ${ }^{1,2}$. Jörg Krämer ${ }^{1} \cdot$ Tim Ratajczyk $^{1}$. \\ Bernhard Maaß ${ }^{1}$. Patrick Müller ${ }^{1}$. Felix Sommer ${ }^{1}$. Wilfried Nörtershäuser ${ }^{1}$
}

Published online: 30 March 2020

(C) The Author(s) 2020

\begin{abstract}
COALA is a new offline collinear laser spectroscopy setup for high-precision measurements and development work at TU Darmstadt, Germany. An introduction to the technique and the experimental setup is given and an overview of current projects with recent results is presented. The idea of a novel all-optical absolute charge radius determination is discussed.
\end{abstract}

Keywords Collinear laser spectroscopy $\cdot$ High precision spectroscopy $\cdot$ High voltage evaluation $\cdot$ Nuclear charge radii

\section{Introduction}

Collinear laser spectroscopy (CLS) is a high-resolution laser spectroscopy technique. The basic principle is the superposition of a laser beam and a beam of fast ions or atoms along the ion-beam direction as shown in Fig. 1. The laser will excite the ions when at resonance with the Doppler-shifted atomic transition frequency. In the simplest version of CLS, the spontaneous emission of the excited atoms is then detected with a photomultiplier tube (PMT) perpendicular to the beam in the fluorescence detection region (FDR). Since the atoms are either co-propagating ( $\mathrm{c}=$ collinear) with or counter-propagating $(\mathrm{a}=$ anticollinear) to the laser beam at the velocity $\beta=v / c$, the transition frequency $v_{0}$ is shifted in the laboratory system by the relativistic Doppler effect according to

$$
v_{\mathrm{c} / \mathrm{a}}=v_{0} \gamma(1 \pm \beta)
$$

This article is part of the Topical Collection on Proceedings of PLATAN 2019, 1st International Conference, Merger of the Poznan Meeting on Lasers and Trapping Devices in Atomic Nuclei Research and the International Conference on Laser Probing, Mainz, Germany 19-24 May 2019

Edited by Krassimira Marinova, Michael Block, Klaus D.A. Wendt and Magdalena Kowalska

Phillip Imgram

pimgram@ikp.tu-darmstadt.de

1 Institut für Kernphysik, Technische Universität Darmstadt, 64289, Darmstadt, Germany

2 Present address: National Superconducting Cyclotron Laboratory, Michigan State University, East Lansing, MI 48824, USA 


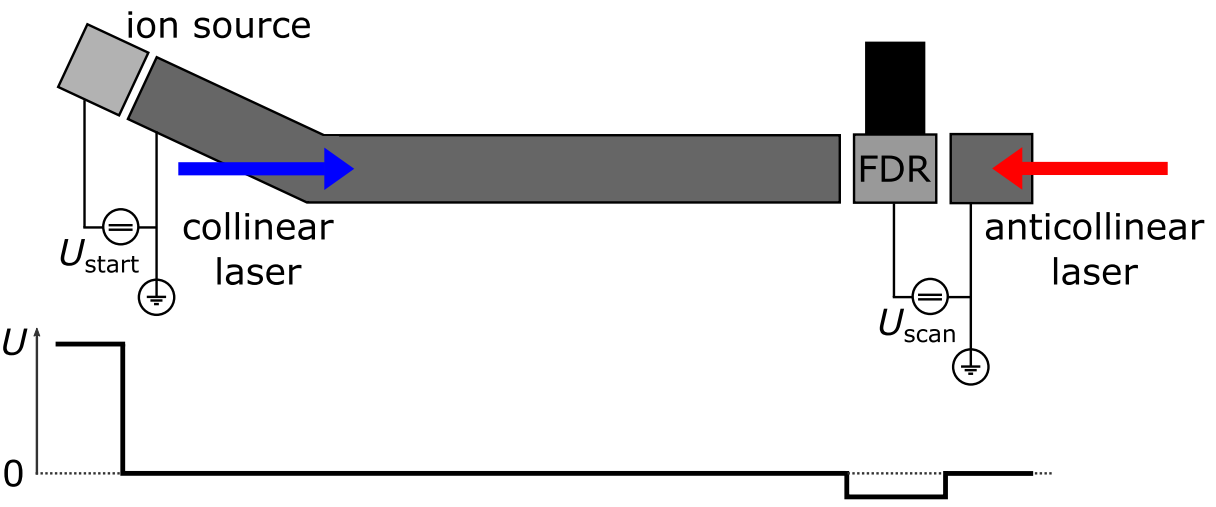

Fig. 1 Simplified scheme of collinear laser spectroscopy. Electrostatically accelerated ions are superposed with a copropagating or counterpropagating laser beam. Fluorescence light is detected with a photo multiplier tube in a floatable fluorescence detection region (FDR). Doppler tuning is performed by changing the voltage at the FDR as indicated

with the Lorentz factor $\gamma=\left(1-\beta^{2}\right)^{-1 / 2}$. Hence, the resonance can be found either by scanning the laser frequency or by manipulating the ion velocity. This opens up the possibility of so-called Doppler-tuning by applying a scannable, electrostatic potential to accelerate or decelerate the ions until they are in resonance with a fixed laser frequency $v_{\mathrm{a} / \mathrm{c}}$. This is usually much easier than scanning the laser since voltages can be ramped much faster and with higher reproducibility than most commercial single-mode laser systems. Even fixedfrequency lasers can be used provided that their wavelength is sufficiently close to the transition wavelength.

The high resolution of CLS originates from the initial acceleration of the ions through an electrostatic potential of the order of typically $15 \mathrm{kV}$ to $60 \mathrm{kV}$. This has two important effects: First, the ionic velocity distribution in the direction of flight is strongly compressed which leads to optical linewidths in the order of the natural linewidth of allowed dipole transitions. Second, it takes only few $\mu s$ from the production of the ions to their investigation enabling the examination of exotic nuclei or metastable ionic states even if they have lifetimes in the ms range.

The connection between atomic spectra and nuclear ground-state properties is provided by the hyperfine splitting and the isotope shift. The former contains information on the nuclear spin, the magnetic dipole and the electric quadrupole moment. The latter includes the change of the mean square charge radius $\delta\left\langle r^{2}\right\rangle^{A}, A^{\prime}$ between two Isotopes $A$ and $A^{\prime}$ in the difference of their respective transition frequencies $v_{i}^{A}$ and $v_{i}^{A^{\prime}}$ in a transition $i$. It is given by

$$
\delta v_{i}^{A, A^{\prime}}=v_{i}^{A^{\prime}}-v_{i}^{A}=K_{i} \frac{M_{A^{\prime}}-M_{A}}{M_{A} M_{A^{\prime}}}+F_{i} \delta\left\langle r_{c}^{2}\right\rangle^{A, A^{\prime}}
$$

with the isotope masses $M_{A}$ and $M_{A^{\prime}}$, the mass shift constant $K_{i}$ and the field shift constant $F_{i}$.

In combination, this allows the survey of nuclear properties with highly accurate optical methods in order to carry out precise tests of nuclear structure theories. This is the main reason for the ongoing success of CLS [1,2] after the first experiments in the 1980s [3]. Although many setups are currently gathered at on-line facilities [2, 4-6], the new offline Collinear Apparatus for Laser Spectroscopy and Applied Science (COALA) has been established at TU Darmstadt, Germany. The primary goal of COALA is the development of 


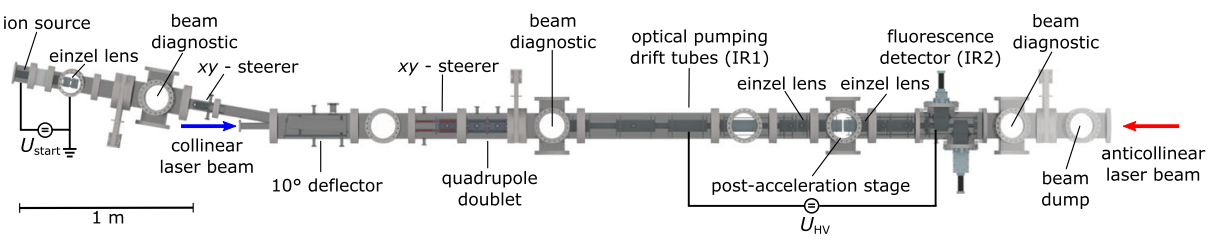

Fig. 2 Full overview of the ultra-high vacuum COALA beamline which is designed for high-precision collinear laser spectrosocpy. Ions with a beam energy of 10-20 keV are collimated and superposed with an incoming laser beam through electrostatic ion optical elements. Resonant fluorescence light of the ions can be detected in the floatable optical detection region (IR2). Drift tubes before IR2 enable optical pumping and manipulation of the ions if needed

new techniques and improvements for CLS. This contribution provides an overview of the COALA setup and some ongoing projects as well as the presentation of recent results.

\section{Experimental setup}

The COALA setup is a very versatile system for high-precision CLS regarding the ion sources and the laser system. A complete and detailed report of the COALA setup is in preparation and will be published soon. Therefore, only a brief overview of the setup is given here.

\subsection{Ultra-high vacuum beamline}

A scheme of the complete beamline is depicted in Fig. 2. Ions from any available source (e.g. surface ionization source, liquid metal ion source etc.) are accelerated through a high voltage into the beamline. Different electrostatic ion-optical elements are used to produce a collimated ion beam which is then superposed with an incoming laser beam. Fluorescence light of the ions can be detected in the FDR with an elliptical mirror system and two photomultiplier tubes (PMTs). Furthermore, the FDR can be floated with respect to the rest of the beamline by up to $20 \mathrm{kV}$ to enable Doppler-tuning. In order to ensure a good superposition of laser and ions the beamline possesses two beam diagnostic stations in front and after the FDR featuring well-aligned iris diaphragms and fluorescence screens. Additionally, 1.2-m long drift tubes in the middle of the setup allow for optical pumping of the ions if needed.

\subsection{Laser setup}

The full laser setup is depicted in Fig. 3 and is based on two titanium-sapphire (Ti:Sa) lasers (Sirah MATISSE 2 TS). If required, both lasers can also be converted to dye lasers opening up an even wider wavelength range. Both laser systems can then be extended with up to two Sirah WaveTrain frequency doubling stages in order to produce deep ultra-violet light. Depending on the wavelength the laser light is then transported to the beamline either in an optical fiber or in free space. Both fundamental lasers are stabilized to scannable reference cavities which are again stabilized to a Menlo Systems FC1500 frequency comb facilitating an absolute frequency stability better than $100 \mathrm{kHz}$. The measurement of the laser frequency is done in parallel with wavemeters and the frequency comb. This combination makes the COALA setup very flexible in the selection of the ion species and transitions. 


\section{The Collinear Apparatus for Laser Spectroscopy and Applied Science (COALA)}

Originally the COALA beamline was developed for the Accurate Laser Involved Voltage Evaluation (ALIVE) experiment. During this endeavor many systematics of the apparatus were well understood and minimized. This enabled the possibility for conventional CLS with very high precision. From that a new project arose which is the determination of absolute charge radii from an all-optical approach, which will be worked on in the coming years.

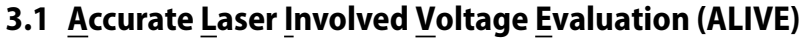

As explained before, the acceleration of the ions of interest gives rise to the relativistic Doppler effect which shifts the rest-frame resonance frequency $v_{0}$ of the fast ions in the laboratory frame and the laser has to be operated at the shifted frequency $v_{\mathrm{L}}$ to be in resonance. This means a potential difference $U$ is directly linked to these frequencies via

$$
U=\frac{m c^{2}}{2 q} \frac{\left(\nu_{0}-v_{\mathrm{L}}\right)^{2}}{v_{0} v_{\mathrm{L}}} .
$$

Thus, a high voltage measurement can be attributed to fundamental constants and a frequency measurement giving the opportunity to set a quantum standard. Since a detailed description of the measurement principle can be found in [7,8] only a brief overview is given here. In the ALIVE experiment a pump-and-probe scheme is used in order to exclude systematic uncertainties originating from the ion source voltage. Generally, that means using one laser to optically pump one specific ion velocity class first, accelerating the ions with the high voltage of interest and probing them with a second laser afterwards. Through comparison of the two laser frequencies which have been used to excite the ions before and after the acceleration, one already achieves a precision of 100 parts per million (ppm) as demonstrated in [9]. In order to improve the high voltage measurements further, the pump-and-probe approach has been expanded with a reference-measurement approach in the ALIVE experiment. Here, a $0 \mathrm{~V}$ measurement is carried out additionally to the high voltage measurement with the same scheme. The difference between both measurements largely reduces systematic uncertainties stemming from contact and thermal potentials and the laser-ion overlap. In the first measurement campaign with ${ }^{40} \mathrm{Ca}^{+}$an accuracy of $5 \mathrm{ppm}$ compared to a calibrated high voltage divider has been demonstrated [7] whereas the world's best high voltage divider reaches $1 \mathrm{ppm}$. In a second step ${ }^{115} \mathrm{In}^{+}$ions from a liquid metal ion source (LMIS) have been used. This looked like a promising candidate first due to the narrow natural linewidth of the ${ }^{1} \mathrm{~S}_{0} \rightarrow{ }^{3} \mathrm{P}_{1}$ intercombination transition and the very good beam emittance of the LMIS. However, time-of-flight broadening and the limited statistics caused by the inefficient pump process in the targeted transition did not yield an accuracy improvement. Nevertheless, the beamline and the laser system have been improved further during that campaign which triggered a new and now ongoing try with ${ }^{40} \mathrm{Ca}^{+}$.

\subsection{Determination of absolute nuclear charge radii}

Conventional CLS usually focuses on the measurements of isotope shifts thus differences of transition frequencies. The reason for that is the relativistic Doppler effect. In order to derive the rest-frame transition frequency from the laboratory frame frequency, a precise knowledge of the ion velocity is required which is usually not the case due to unknown starting potentials of the ions and contact potentials. Therefore, even small deviations from 


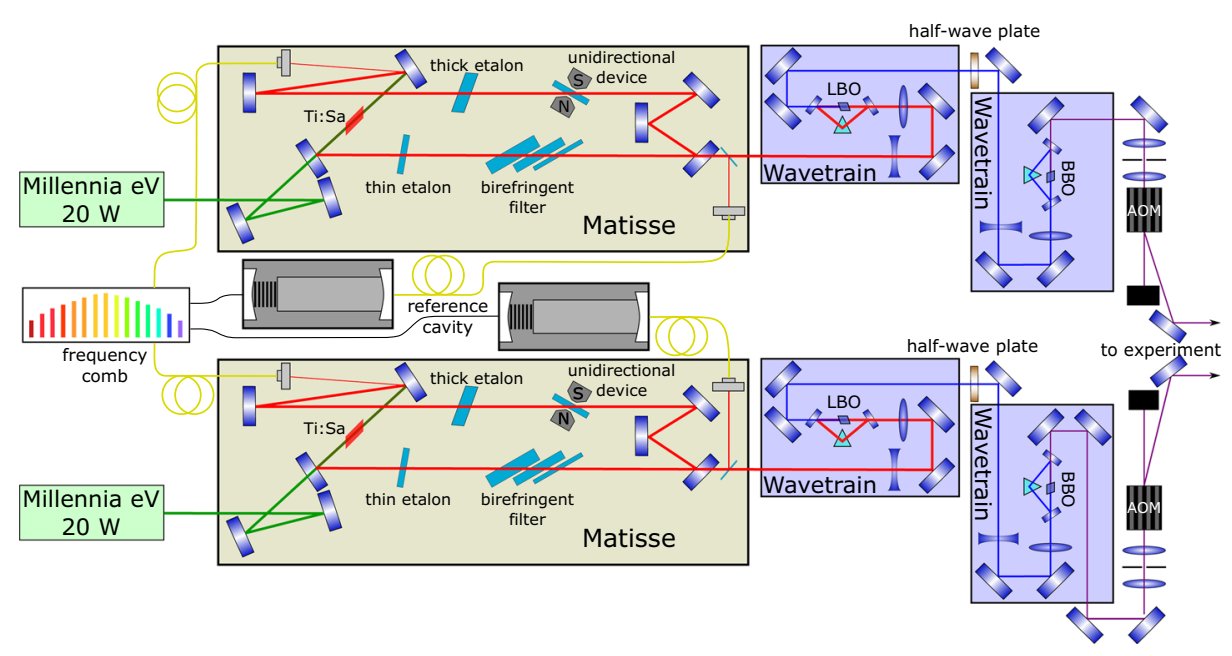

Fig. 3 Full laser setup consisting of two identical laser systems. One system consists of a $20 \mathrm{~W}$ SpectraPhysics Millennia eV pumped continuous-wave titanium:sapphire Sirah Matisse 2 TS laser. Afterwards, UV light can be produced down to about $200 \mathrm{~nm}$ with two Sirah WaveTrain frequency doubling stages. The produced light can then be transported via optical fiber or free space to the beamline depending on the wavelength

the exact parallel geometry of laser and ion beam will introduce rather large errors. However, using a copropagating and a counterpropagating laser (quasi-)simultaneously cancels out the Doppler-shift by multiplying both laboratory frame frequencies

$$
v_{\mathrm{c}} v_{\mathrm{a}}=v_{0}^{2} \gamma^{2}(1+\beta)(1-\beta)=v_{0}^{2}
$$

and makes the rest-frame transition frequency $\nu_{0}$ accessible for CLS as well [10,11]. This is interesting not only for atomic but also for nuclear physics since $v_{0}$ also contains the "full" nuclear size $\left\langle r_{\mathrm{c}}^{2}\right\rangle$ in the finite-size effect

$$
v_{0}=v_{\text {point }}-\frac{Z e^{2}}{6 h \varepsilon_{0}} \Delta\left|\Psi_{\mathrm{e}}(0)\right|_{\mathrm{i} \rightarrow \mathrm{f}}^{2} \cdot\left\langle r_{\mathrm{c}}^{2}\right\rangle=v_{\text {point }}+F_{\mathrm{i} \rightarrow \mathrm{f}} \cdot\left\langle r_{\mathrm{c}}^{2}\right\rangle
$$

where $v_{\text {point }}$ is the transition frequency (calculated) for a point-like nucleus and $\Delta\left|\Psi_{\mathrm{e}}(0)\right|_{\mathrm{i} \rightarrow \mathrm{f}}^{2}$ is the change of the electron density at the nucleus in the transition $\mathrm{i} \rightarrow \mathrm{f}$. Now, the basic idea is to investigate a system where $v_{\text {point }}$ and $F_{\mathrm{i} \rightarrow \mathrm{f}}$ can be calculated to sufficient precision. Together with the experimental value for $v_{0}$ the absolute nuclear mean-square charge radius $\left\langle r_{\mathrm{c}}^{2}\right\rangle$ can then be extracted. This approach has already been used for hydrogen and muonic hydrogen. Unfortunately, the atomic structure calculations beyond hydrogenlike systems are very demanding but a program for He-like systems has been started [12] and results are expected in the near future. Laser spectroscopy cannot be easily performed from the groundstate in He-like systems, since the energy gap to the next energy level is in the order of hundreds of $\mathrm{eV}$. Instead, transitions from the meta-stable $1 s 2 s^{3} \mathrm{~S}_{1}$ state are considered. Multiply charged, He-like ions in this state can be produced with an electron beam ion source (EBIS) where around $10 \%$ of the produced ions in the correct charge state are expected to be in the ${ }^{3} \mathrm{~S}_{1}$ state. However, the lifetime of this state rapidly decreases with increasing

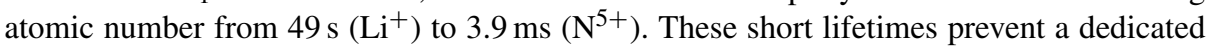
examination in ion traps due to their relatively slow cooling process. In parallel to that the excitation wavelength for the lowest-lying $1 s 2 s{ }^{3} \mathrm{~S}_{1} \rightarrow 1 s 2 p{ }^{3} \mathrm{P}_{J}$ transition decreases as 


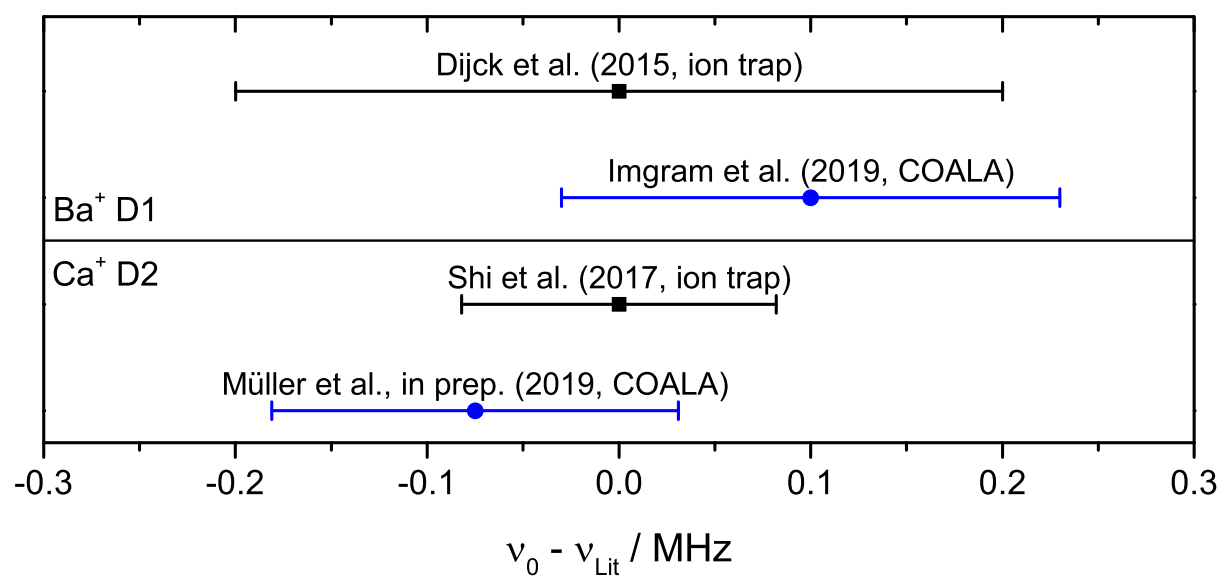

Fig. 4 Recent COALA results for rest-frame transition frequencies compared to ion trap measurements (Dijck et al. [13], Shi et al. [15]). Both measurements show that COALA measurements have an accuracy comparable to the ion trap results. Naturally, collinear laser spectroscopy is not competitive in accuracy for forbidden transitions, which can be determined in ion traps with many orders of magnitude higher accuracy

well from $550 \mathrm{~nm}\left(\mathrm{Li}^{+}\right)$to $190 \mathrm{~nm}\left(\mathrm{~N}^{5+}\right)$ and becomes unreachable with continuous-wave lasers for all heavier nuclei beyond. Summarized, a fast and precise technique is needed in order to extract absolute nuclear charge radii from an all-optical approach making CLS an optimal choice. A rough estimation using scaling laws shows that an accuracy of $1 \mathrm{MHz}$ in the experiment would lead to an accuracy of about $0.25 \%$ in $\left\langle r_{\mathrm{c}}^{2}\right\rangle$. To demonstrate the precision that can be reached with (quasi-)simultaneous collinear and anticollinear spectroscopy, stable $\mathrm{Ba}^{+}$ions have been investigated in the $6 s^{2} S_{1 / 2} \rightarrow 6 p^{2} P_{J}$ doublet due to a precise existing measurement of ${ }^{138} \mathrm{Ba}^{+}$in an ion trap [13]. The complete results of this measurement can be found in [14]. In brief, it has been shown that the CLS result matches the ion trap result in full agreement and even improved the uncertainty by almost a factor of 2 to about $130 \mathrm{kHz}$. This is illustrated in Fig. 4 together with a preview of a recent investigation in $\mathrm{Ca}^{+}$which was the next step towards lighter nuclei. Also there the accuracy of the COALA measurement is comparable to the ion trap results. A dedicated publication for the $\mathrm{Ca}^{+}$campaign will follow soon. The reason for the step-by-step approach towards lighter nuclei is the increasing differential Doppler-shift $\frac{\partial v}{\partial U}$ with decreasing mass leading to growing systematic uncertainties. Additionally, this factor increases even more rapidly for higher charge states. Therefore a careful analysis of the experimental setup had been carried out in a regime where reference measurements are available like for $\mathrm{Ba}^{+}$[13] and $\mathrm{Ca}^{+}$[15]. Additionally, these investigations have been of interest from a physics point of view due to the " $\mathrm{Ca}^{+}$field-shift puzzle" as reported in [15].

\section{Summary and Outlook}

In the last years the capabilities of COALA have been demonstrated. The high-voltage evaluation is already in reach of the world's best high-voltage dividers with an accuracy of $5 \mathrm{ppm}$. Further improvements such as a fast and precise laser scanning scheme and a high voltage stabilization scheme are currently under development. This will not only help the 
ALIVE experiment to reach the $1 \mathrm{ppm}$ but also to establish a high accuracy for the restframe transition frequency determination in He-like light ions. Furthermore, first tests with negative ions are carried out at the moment. The idea here is to neutralize the negative ions with a high-repetition pulsed laser in order perform collinear laser spectroscopy on atoms without a charge exchange cell. This might broaden the spectrum of possible experiments at COALA further.

Acknowledgements Open Access funding provided by Projekt DEAL. We acknowledge financial support from the German Federal Ministry for Education and Research (BMBF) under Contract No. 05P15RDFN1, the Helmholtz International Center for FAIR and funding by the Deutsche Forschungsgemeinschaft (DFG) Project No. 279384907 SFB 1245. Installation of COALA was supported by the DFG under Grant INST No. 163/392-1 FUGG. P.I., K.K, T.R., B.M. and F.S. acknowledge support from HGS-HIRE.

Open Access This article is licensed under a Creative Commons Attribution 4.0 International License, which permits use, sharing, adaptation, distribution and reproduction in any medium or format, as long as you give appropriate credit to the original author(s) and the source, provide a link to the Creative Commons licence, and indicate if changes were made. The images or other third party material in this article are included in the article's Creative Commons licence, unless indicated otherwise in a credit line to the material. If material is not included in the article's Creative Commons licence and your intended use is not permitted by statutory regulation or exceeds the permitted use, you will need to obtain permission directly from the copyright holder. To view a copy of this licence, visit http://creativecommonshorg/licenses/by/4.0/.

\section{References}

1. Blaum, K., Dilling, J., Nörtershäuser, W.: Precision atomic physics techniques for nuclear physics with radioactive beams. Physica Scripta T152, 014017 (2013). https://doi.org/10.1088/0031-8949/2013/ t152/014017

2. Campbell, P., Moore, I.D., Pearson, M.R.: Laser spectroscopy for nuclear structure physics. Progress in Particle and Nuclear Physics 86, 127-180 (2016). https://doi.org/10.1016/j.ppnp.2015.09.003. http:// www.sciencedirect.com/science/article/pii/S0146641015000915

3. Neugart, R.: Nuclear Instruments and Methods in Physics Research 186(1), 165 (1981). https://doi.org/10.1016/0029-554X(81)90902-2. http://www.sciencedirect.com/science/article/pii/00295 $54 X 81909022$

4. Neugart, R., Billowes, J., Bissell, M.L., Blaum, K., Cheal, B., Flanagan, K.T., Neyens, G., Nörtershäuser, W., Yordanov, D.T.: Journal of Physics G: Nuclear and Particle Physics 44(6), 064002 (2017). https://doi.org/10.1088/1361-6471/aa6642

5. Minamisono, K., Mantica, P., Klose, A., Vinnikova, S., Schneider, A., Johnson, B., Barquest, B.: Nuclear instruments and methods in physics research section a: accelerators, spectrometers, detectors and associated equipment 709, 85 (2013). https://doi.org/10.1016/j.nima.2013.01.038. http://www.sciencedirect. com/science/article/pii/S0168900213001034

6. Ullmann, J., Andelkovic, Z., Brandau, C., Dax, A., Geithner, W., Geppert, C., Gorges, C., Hammen, M., Hannen, V., Kaufmann, S., König, K., Litvinov, Y.A., Lochmann, M., Maaß, B., Meisner, J., Murböck, T., Sánchez, R., Schmidt, M., Schmidt, S., Steck, M., Stöhlker, T., Thompson, R.C., Trageser, C., Vollbrecht, J., Weinheimer, C., Nörtershäuser, W.: Nat. Commun. 8(1), 15484 (2017). https://doi.org/10.1038/ncomms15484

7. Krämer, J., König, K., Geppert, Ch., Imgram, P., Maßß, B., Meisner, J., Otten, E.W., Passon, S., Ratajczyk, T., Ullmann, J., Nörtershäuser W.: High-voltage measurements on the 5 ppm relative uncertainty level with collinear laser spectroscopy. Metrologia 55(2), 268 (2018). http://stacks.iop.org/0026-1394/ $55 / \mathrm{i}=2 / \mathrm{a}=268$

8. König, K., Geppert, C., Krämer, J., Maaß, B., Otten, E.W., Ratajczyk, T., Nörtershäuser, W.: Hyperfine Interact. 238(1), 24 (2017). https://doi.org/10.1007/s10751-016-1392-4

9. Will, E., Ullmann, J., Frömmgen, N., Geppert, C., Gorges, C., Hammen, M., Kaufmann, S., Krieger, A., Nörtershäuser, W.: Hyperfine Interact. 227(1), 157 (2014). https://doi.org/10.1007/s10751-014-1053-4

10. Krieger, A., Blaum, K., Bissell, M.L., Frömmgen, N., Geppert, C., Hammen, M., Kreim, K., Kowalska, M., Krämer, J., Neff, T., Neugart, R., Neyens, G., Nörtershäuser, W., Novotny, C., Sánchez, R., Yordanov, D.T.: Phys. Rev. Lett. 108, 142501 (2012). https://doi.org/10.1103/PhysRevLett.108.142501 
11. Krieger, A., Nörtershäuser, W., Geppert, C., Blaum, K., Bissel, M.L., Frömmgen, N., Hammen, M., Kreim, K., Kowalska, M., Krämer, J., Neugart, R., Neyens, G., Sanchez, R., Tiedemann, D., Yordanov, D., Zakova, M.: Appl. Phys. B 123, 15 (2017)

12. Yerokhin, V.A., Patkóš, V.c.v., Pachucki, K.: Phys. Rev. A 98, 032503 (2018). https://doi.org/10.1103/ PhysRevA.98.032503

13. Dijck, E.A., Nuñez Portela, M., Grier, A.T., Jungmann, K., Mohanty, A., Valappol, N., Willmann, L.: Phys. Rev. A 91, 060501 (2015). https://doi.org/10.1103/PhysRevA.91.060501

14. Imgram, P., König, K., Krämer, J., Ratajczyk, T., Müller, R.A., Surzhykov, A., Nörtershäuser, W.: Phys. Rev. A 99, 012511 (2019). https://doi.org/10.1103/PhysRevA.99.012511

15. Shi, C., Gebert, F., Gorges, C., Kaufmann, S., Nörtershäuser, W., Sahoo, B.K., Surzhykov, A., Yerokhin, V.A., Berengut, J.C., Wolf, F., Heip, J.C., Schmidt, P.O.: Appl. Phys. B 123(1), 2 (2016). https://doi.org/10.1007/s00340-016-6572-Z

Publisher's note Springer Nature remains neutral with regard to jurisdictional claims in published maps and institutional affiliations. 